Perspective

\title{
A Framework for Evaluating and Disclosing the ESG Related Impacts of AI with the SDGs
}

\author{
Henrik Skaug Sætra
}

check for updates

Citation: Sætra, H.S. A Framework for Evaluating and Disclosing the ESG Related Impacts of AI with the SDGs. Sustainability 2021, 13, 8503. https://doi.org/10.3390/su13158503

Academic Editors: Aimee

van Wynsberghe, Larissa Bolte and Jamila Nachid

Received: 29 May 2021

Accepted: 28 July 2021

Published: 29 July 2021

Publisher's Note: MDPI stays neutral with regard to jurisdictional claims in published maps and institutional affiliations.

Copyright: (C) 2021 by the author. Licensee MDPI, Basel, Switzerland. This article is an open access article distributed under the terms and conditions of the Creative Commons Attribution (CC BY) license (https:// creativecommons.org/licenses/by/ $4.0 /)$.
Faculty of Business, Languages, the Social Sciences, Østfold University College, N-1757 Halden, Norway; Henrik.satra@hiof.no

\begin{abstract}
Artificial intelligence (AI) now permeates all aspects of modern society, and we are simultaneously seeing an increased focus on issues of sustainability in all human activities. All major corporations are now expected to account for their environmental and social footprint and to disclose and report on their activities. This is carried out through a diverse set of standards, frameworks, and metrics related to what is referred to as ESG (environment, social, governance), which is now, increasingly often, replacing the older term CSR (corporate social responsibility). The challenge addressed in this article is that none of these frameworks sufficiently capture the nature of the sustainability related impacts of AI. This creates a situation in which companies are not incentivised to properly analyse such impacts. Simultaneously, it allows the companies that are aware of negative impacts to not disclose them. This article proposes a framework for evaluating and disclosing ESG related AI impacts based on the United Nation's Sustainable Development Goals (SDG). The core of the framework is here presented, with examples of how it forces an examination of micro, meso, and macro level impacts, a consideration of both negative and positive impacts, and accounting for ripple effects and interlinkages between the different impacts. Such a framework helps make analyses of AI related ESG impacts more structured and systematic, more transparent, and it allows companies to draw on research in AI ethics in such evaluations. In the closing section, Microsoft's sustainability reporting from 2018 and 2019 is used as an example of how sustainability reporting is currently carried out, and how it might be improved by using the approach here advocated.
\end{abstract}

Keywords: artificial intelligence; Sustainable Development Goals; ESG; CSR; reporting; disclosure

\section{Introduction}

Artificial intelligence (AI) permeates all aspects of modern society, and the forthcoming artificial intelligence revolution is arguably already here [1]. Both businesses and governments now implement AI systems on a large scale, both to proactively gain benefits and in fear of being left behind as others do so [2-4].

Simultaneously, businesses, civil society, politicians, and regulators are increasingly focusing on the sustainability of all human activity [5]. All major corporations are now expected to understand their environmental and social footprint and to disclose and report on related activities. This is carried out through a diverse set of standards, frameworks, and metrics related to what is referred to as ESG (environment, social, governance), which is now, increasingly often, replacing the older term CSR (corporate social responsibility) [6].

The world of AI and big tech is not exempt from these expectations, and the challenge addressed in this article is that none of the existing ESG standards, frameworks, or metrics sufficiently capture the nature of the sustainability related impacts of AI. This creates a situation in which companies are not incentivised to properly analyse and evaluate such impacts. Simultaneously, it allows the companies that are aware of negative impacts to not disclose them.

This article focuses on the need to implement insights from AI ethics research in ESG reporting, and emphasizes how the Sustainable Development Goals (SDGs) can be used to 
evaluate and categorize the potential sustainability related benefits and harms related to AI [7]. The SDGs encompass environmental goals, goals related to social justice, and goals related to economic growth, health and work. This makes the SDGs useful for analyzing the sustainability of AI, which includes both how AI is used for sustainable activities, and how the use of AI might simultaneously have, for example, negative environmental impacts [8].

The outlines of a framework and a process for evaluating and disclosing ESG related AI impacts by using the SDGs are here presented, with a particular emphasis on how businesses are increasingly both expected to and required to report on and disclose such information. Examples of how the framework can force an examination of micro, meso, and macro level impacts are provided. In addition, it is shown how the framework is used to promote a consideration of both negative and positive impacts, while also accounting for ripple effects and interlinkages between the different impacts. Such a framework would make analyses of AI related ESG impacts more structured and systematic, more transparent, and allow companies to draw on research in AI ethics in such evaluations. The framework can be used by large businesses-whose primary business is the production and application of AI systems - and all others that use AI systems in parts of their activities. A comprehensive evaluation of the specific impacts of AI systems on all goals and subgoals is beyond the scope of this article, but the general approach to using the SDGs will be demonstrated through a set of examples and an examination of how Microsoft describes their AI related ESG impacts.

First of all, the concept of sustainability is examined in Section 2, with a particular focus on how it is embodied in the SDGs and how various ESG standards, frameworks, and metrics have been developed in order to help companies understand and communicate the sustainability of their activities. Secondly, in Section 3, I provide a basic account of the potential linkages between AI and environmental, social, and governance related risks and impacts in order to establish why these impacts should be taken more seriously and why it is a problem that they are partly neglected in major ESG related frameworks. Finally, the use of the SDGs in working with sustainability evaluation and disclosure is discussed in Section 4, along with the general outline of the proposed framework. In closing, selected sustainability reports from Microsoft are used to demonstrate how AI related impacts have been communicated historically, and how the framework here proposed might improve the situation.

\section{Sustainability, the SDGs, and Efforts to Tackle the Sustainability of Corporations}

The concept of sustainability might appear to be both intuitive and relatively straightforward. Sustainability, and the idea of sustainable development, is usually traced back to the 1987 report Our Common Future produced by a United Nations (UN) commission headed by Gro Harlem Brundtland. Today, sustainability in the context of business and politics is increasingly often aligned with the SDGs. These goals were presented by the UN in the document Transforming our World: The 2030 Agenda for Sustainable Development [7]. The framework consists of the 17 goals shown in Figure 1. With the SDGs, the notion of sustainability and a broad perspective of societal and human development on a global scale are thoroughly intertwined. This is in line with the early work on sustainable development carried out by Brundtland et al. [9]. While the SDGs are clearly related to a range of basic human rights, the two frameworks are clearly distinct, as the SDGs entail a broader focus on what is referred to as the five P's: people, planet, prosperity, peace, and partnership [7]. 

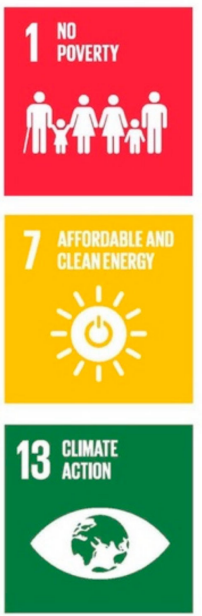
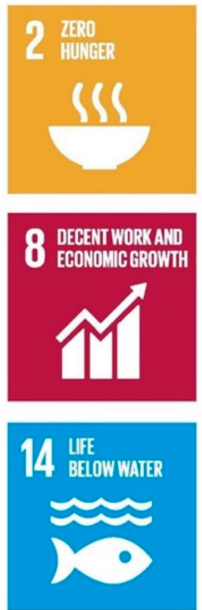
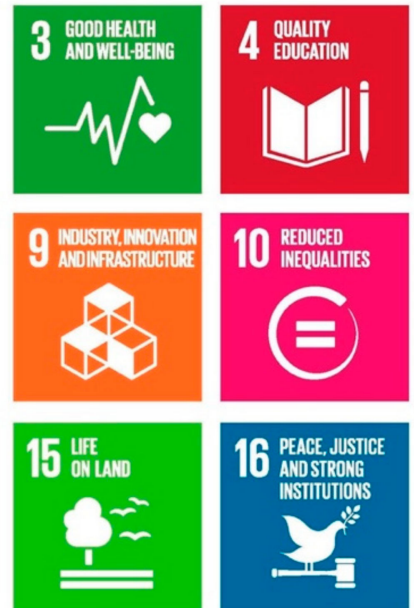
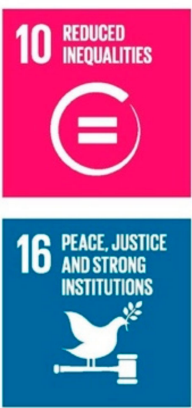
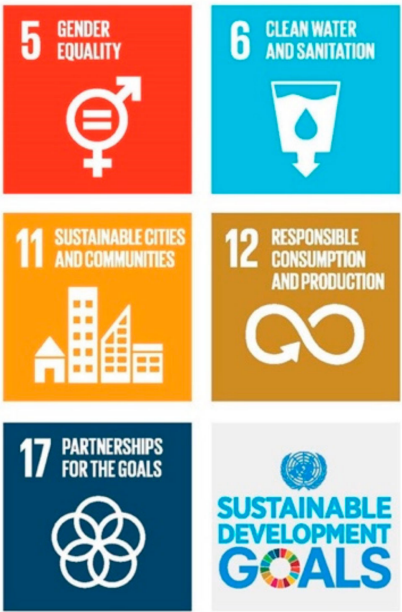

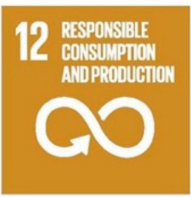

(8) SUSTAINABLE DEVELOPMENT GOALS

Figure 1. The Sustainable Development Goals [7].

While development and environmental protection might be thought of as a task for governments, the world of business and finance is increasingly playing an active role in reaching the SDGs. In part through coercion in the form of law and regulation, but also in part due to more informal processes related to the need to secure a social license to operate $[6,10]$. Investors, business partners, and customers now tend to demand more in terms of corporate responsibility than that which is required by law alone. This leads to a situation in which sustainability is part compliance (a "hygiene factor") and part proactive strategic business development, as sustainable business models are increasingly shown to provide businesses with an advantage in the marketplace [6,11-13]. The responsibilities of corporations to contribute to sustainable development, or at the very least to disclose how their activities have sustainability impacts, is a key part of the question examined in this article.

Corporate social responsibility (CSR) is nothing new [11], and a 40 year old quote describes fairly well the current challenges that have led to an increased focus on sustainability:

There are several reasons for the current concern with corporate social responsibility. In recent years, the level of criticism of the business system has risen sharply. Not only has the performance of business been called into question, but so too have the power and privilege associated with large corporations. Some critics have even questioned the corporate system's ability to cope with future problems. [14] (p. 59)

Today, issues of privilege, social justice, and the societal and environmental consequences of businesses are more relevant than ever before. As a consequence, the term CSR now refers to a much broader set of issues than it did in the early ages of the concept [6]. Environmental challenges related to a range of issues, such as climate change, biodiversity loss, pollution, etc., are now universally recognized as a key challenge for humanity. In addition, as this article emphasizes the impact of AI, the unprecedented power and influence of the major technology companies attracts both attention and scrutiny. Big Tech describes the major tech companies, and GAFAM is one acronym describing the major US players: Google, Amazon, Facebook, Apple, and Microsoft (MS) [15]. A host of issues related to the activities of these companies are debated, and amongst the most prominent are their close to monopoly power [15], and their use of surveillance and data to monitor and increasingly exert influence over individuals and society [16]. Ethical issues more closely related to AI systems are discussed in Section 4, where it is shown how both environmental, social, and governance related risks must be mapped in order to fully understand the impact of AI.

While many focus on the three dimensions of sustainability (society, economy, and environment) when discussing the SDGs [7,17], this article focuses specifically on the 
evaluation and disclosure of AI impacts, and in this context it can be useful to align the SDGs with the three aspects of ESG. Figure 2 shows a figure inspired by Berenberg [18]. The framework here presented is primarily intended as a tool for evaluating and fostering understanding of the actual impacts of AI systems, and the results from using the framework can be presented in a number of ways, as will be shown in Section 4.

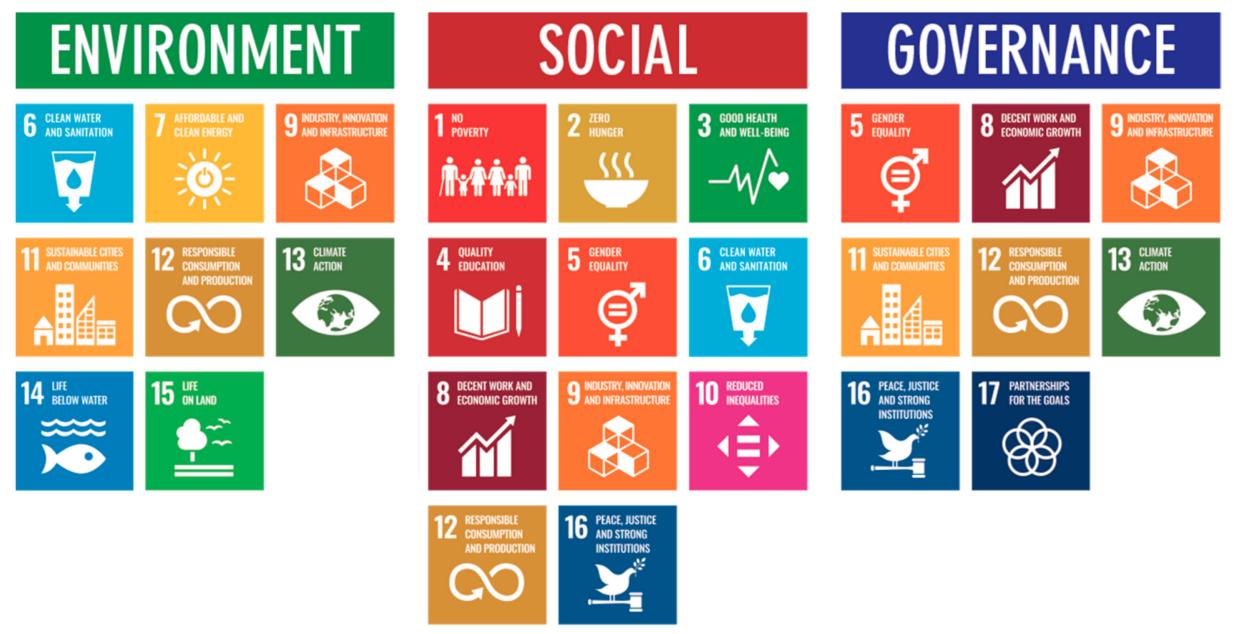

Figure 2. SDG through the lens of ESG [18].

While the proposed framework might be useful for analyzing AI impacts in general, this article focuses on how corporations can analyze such impacts in order to both understand and communicate them. AI ethics research more generally deals with the same kinds of impacts, but the main challenges addressed here are the problems of making use of the insight created by such research when analyzing and reporting on corporate sustainability. It is, in part, a framework that helps translate research to a business setting.

Different types of companies in different regions all face different demands for disclosure and reporting, and in relation to disclosure requirements, ESG is the term now most often used [19]. In terms of financial regulation, for example, the Securities and Exchange Commission (SEC) in the US leaves it up to companies to determine what is material information to be disclosed, while European authorities are implementing mandatory obligations enforced by individual countries [20]. The European Union's (EU) Green Deal [21] with the sustainable finance initiative and the related taxonomy [22], which is a classification system for determining the sustainability of various economic activities, serves to illustrate how seriously regulators in Europe now take ESG related disclosure and risks. While the formal requirements for the financial sector are most developed, the trend in all sectors and businesses is clear: stakeholders of all kinds demand information about the sustainability impact and risks of business activities. Efforts to streamline and make the disclosure of such information comparable, universal, and accessible are thus increasingly relevant for all types of businesses.

As a consequence of these developments, a range of different standards and frameworks for disclosure and reporting on ESG have been developed, and this is now a landscape often described as an "alphabet soup," marred by a dizzying array of choices and few clear guidelines from regulators. Some of the major standards and frameworks mentioned in this article are the Global Reporting Initiative (GRI), the Sustainability Accounting Standards Board (SASB), the World Economic Forum's (WEF) Stakeholder Capitalism Metrics (SCM), and the SDGs. A detailed examination of the full range of standards and frameworks is beyond the scope of this chapter. Other important standards and frameworks such as the Carbon Disclosure Project (CDP) and Task Force on Climate-Related Financial Disclosures (TCFD), and more detailed examinations of GRI, SASB and the SDGs are found in [23]. These are mentioned because they are currently amongst the most popular choices 
for businesses. There are many others as well, but the specifics of all these frameworks are not what is important here. What is important is that they are all insufficient in terms of incentivizing businesses to evaluate and disclose AI produced sustainability impacts.

GRI is the most widely adopted standard for preparing nonfinancial disclosures, often in the form of sustainability reports, and it can be used in combination with other frameworks [23]. While the GRI focuses on a wide range of stakeholders, the SASB focuses more specifically on investors as the target audience [23]. The WEF stakeholder metrics was designed as a unifying minimal framework that unites and draws upon a range of other frameworks [24].

Regarding the SDGs, they were, at the outset, not intended as an ESG reporting framework, but they are now increasingly used for this purpose [23]. A basic idea behind using the SDGs in such a manner is that they highlight the power of investors and businesses to engender change. The GRI, the UN Global Compact and the World Business Council for Sustainable Development have together developed the SDG Compass to help with the use of the SDGs for reporting (and other business oriented purposes) [25].

The SDGs are emphasized in the following, but mostly related to how they allow for improved analyses and understanding of the ESG related impacts of AI, and not as a replacement for frameworks such as GRI and SASB. This article is thus not a criticism of any particular framework or standard, but rather a call for them to be complemented by a more specific framework for analyzing how the use of AI systems, which are now ubiquitous, affect the sustainability of a company. The need for such a complementary framework arises because the other frameworks are created to account for traditional economic activity, while AI systems create certain novel challenges that require specific attention.

\section{The Sustainability Impacts of AI}

A growing number of sources explore the relationship between AI and the SDGs. Some attempts have been made to evaluate how AI relates to all the SDGs $[17,26,27]$. Others have provided more focused analyses of AI and of particular topics or specific SDGs. Some examples are research emphasizing finance related issues [28], and the technological aspects of AI [29]. Others again focus on AI and various issues of sustainability in general, without connecting this to the SDGs [30,31]. Research on sustainable business models, for example, is most often related to sustainability in general [12,13,30], while some do connect it specifically to the SDGs [3]. Efforts to examine the ethical and social implications of AI, for example work on responsible AI [32] and AI4People [33], are also clearly related to the social and governance related SDGs.

Of particular interest, however, are initiatives like the UN initiated AI4Good [34], aimed at using AI to accelerate work on the SDGs. More recently, some have used the term AI for social good (AI4SG) to describe work on AI aimed at the SDGs [35]. However, the phrase is also used by the industry and others in ways that relate it to traditional CSR and general AI ethics [36,37]. These are indications of another alphabet soup in the making, by those seeking to brand new varieties of socially responsible AI.

With AI being the main focus of the article, it is necessary to define what sort of technologies are referred to as AI. A broad and nontechnical definition is beneficial for securing that all relevant impacts are accounted for when evaluating the impact of AI systems, and Vinuesa, Azizpour, Leite, Balaam, Dignum, Domisch, Felländer, Langhans, Tegmark and Nerini [17] (p. 1) provide a description of what constitutes AI:

... we considered as AI any software technology with at least one of the following capabilities: perception-including audio, visual, textual, and tactile (e.g., face recognition), decision-making (e.g., medical diagnosis systems), prediction (e.g., weather forecast), automatic knowledge extraction and pattern recognition from data (e.g., discovery of fake news circles in social media), interactive communication (e.g., social robots or chat bots), and logical reasoning (e.g., theory development from premises). This view encompasses a large variety of subfields, including machine learning. 
There are many potential ways to approach the evaluation of AI impacts in an ESG context, and this article highlights the usefulness of using the SDGs. Different frameworks are made for different purposes, and while these differences might at times be important, at other times the frameworks are more complementary than competitive. For example, the GRI framework is widely used for sustainability reporting, and consists of different standards, with varying specificity, with regard to the detail of disclosure and reporting [38]. The purpose the GRI standards are described as follows:

Sustainability reporting, as promoted by the GRI Standards, is an organisation's practice of reporting publicly on its economic, environmental, and/or social impacts, and hence its contributions-positive or negative-towards the goal of sustainable development. [38] (p. 3)

This is in alignment with the purpose of this article, which is to present an approach to the evaluation of the positive and negative AI impacts to sustainable development. Similarly, the purpose of the new WEF metrics is to enable the measuring of sustainable value creation, and the metrics are explicitly linked to the GRI framework as well as to other relevant frameworks and standards [24]. However, these frameworks are designed for broad and general applications, and their origin is the need of investors to understand the risks of business. These indicators are important for generating a business level snapshot of companies, and they are intended to be generic enough for readers of the result to be able to compare different businesses. While highly useful for securing some basic and comparable basis of evaluating companies, they are not fine masked enough to ensure that all relevant AI related risks are accounted for, as discussed in more detail below.

One major benefit of the SDG framework is its origin and backing by the UN, and the fact that it is aimed at a much broader audience than the more business specific standards and frameworks. While other frameworks offer more specific guidance in the form of indicators and metrics that relate to the particular demands of regulators and, for example, the financial industry, the SDGs can be seen as the broader sustainability aspirations of the global community of business, politics, and civil society. This, and the fact that it was developed in partnership with the business and finance community, has made the SDGs a well known and widely accepted tool. As one purpose of ESG reporting is to inform and create trust between stakeholders and businesses, this is a major advantage of the SDGs.

The SDGs are also very broad, which enables us to evaluate most ethical challenges and the positive potential of AI by relating them to the SDGs. This allows for analyses where the challenges and benefits of AI systems can be identified first, followed by an analysis of how these relate to, for example, specific GRI indicators. In order to embark on their ESG reporting and disclosure journey, a company could map the various ways in which their activities positively and negatively impacted the SDGs, and when accompanied by a materiality analysis, such a preprocess would provide the companies with a more complete picture of their ESG related impacts, including those that are not naturally captured by producing a WEF or GRI report, for example.

A different approach is to start with the metrics, extract the required data (and only this), and then examine whether or not the business is in compliance with various requirements and expectations. While this can also be beneficial, it is not conducive to uncovering AI related sustainability impacts, which is why I here propose a complementary framework. The first approach allows for the examination of a broader set of questions and, not least, for uncovering unexpected and novel benefits and challenges that are not necessarily related to the various indicators found in the general ESG frameworks.

Furthermore, the more specific standards are quite restrictive as they aim to provide uniform and efficient ways to report on ESG related activities. This will, at times, have unfortunate results. For example, the Sustainability Accounting Standards Board (SASB), when discussing which factors were material to technology companies, stated that "business ethics issues are not likely to be material for the technology and communications sector" [23] (p. 26). As the discussion of AI ethics below clearly shows, business ethics is at the very core of the evaluation of AI impacts. However, the SASB Materiality 
Map (https: / / materiality.sasb.org, accessed on 15 February 2021) does indicate that issues related to energy management, customer privacy, data security, employee engagement, diversity \& inclusion, product design and lifecycle management, materials sourcing and efficiency, and competitive behavior are likely to be material for business in the technology and communications sector. These are indeed material issues for businesses developing or using AI systems, but as we will see below, an even more fine masked framework is required for capturing the less obvious potential impacts of AI.

While the SDGs are proposed as a starting point for identifying the impacts, companies can easily translate and use the results in regular sustainability reports if they choose to report according to, for example, GRI or SASB. This is both encouraged and expected, and the GRI has even released a comprehensive guide that links the GRI standard to all the SDG goals and targets [39].

\section{Using the SDGs to Evaluate AI System Impacts}

It now remains to examine the foundations of a framework for evaluating and reporting on sustainability related AI impacts. Some of the recent attempts to link AI and the SDGs indicate that the positive potential of AI is great [17]. This is particularly the case if one examines the various use cases that seemingly connect in some way to either the 17 SDGs or some of the targets $[17,26]$. In the context of ESG related impacts, both the use of AI for sustainable activities and the sustainability of AI systems are relevant [8].

AI systems enable the effective analysis Big Data and this combination can be used in a variety of ways that can potentially aid in the achievement of the SDGs. A basic example would be the implementation of AI in all stages of an enterprise resource planning system (ERP), enabling more effective production, better allocation of human resources, better financing decisions, etc. For example, AI can be used to increase energy efficiency, the effective utilization of resources, and make waste management more effective [40], or to produce product life cycle assessments by predicting energy and environmental impacts [41], all potentially leading to more sustainable businesses and economic activities more in line with circular economic principles and conducive to reduced climate gas emissions. MS, for example, emphasizes the potential of its multitiered Azure, "Power" apps and 365 ecosystems to, amongst many other things, deliver better energy efficiency, better monitoring of glaciers, and using AI to better understand climate risks [42]. Google similarly emphasizes the potential of using its Cloud and broader ecosystem of services to leverage AI for social good [43], and they have also started an "AI Impact challenge" where grants are provided to entrepreneurs using AI to achieve positive impact [44].

All types of businesses might be made more effective, but also governments, cities, and civil society might benefit from insights derived from AI analysis [45]. AI is already used in a wide array of political settings [2], as the benefits of automatic classification and prediction can make a wide range of political and bureaucratic decision-making processes more effective. According to Vinuesa, Azizpour, Leite, Balaam, Dignum, Domisch, Felländer, Langhans, Tegmark and Nerini [17], AI can enable 134 SDG targets, and AI is argued to be extremely effective at enabling SDG 1, 4, 6, 7, 9, 11, 14 and 15.

However, such analyses of AI impacts do not resonate particularly well with the current debates in the field of AI ethics, in which social, governance related, and environmental challenges are analyzed. For example, the use of AI to remedy diversity challenges by removing humans from recruitment processes [46] would be met with dire warnings from all those who emphasize the endemic nature of bias and various forms of human influence over AI systems [47-49]. Attracting most attention from AI ethicists are perhaps the social challenges, including bias in AI systems, the increased use of surveillance and a lack of privacy [50] as well as using the combination of AI and Big Data to influence and manipulate $[51,52]$. Such issues are arguably very difficult to map to the indicators covered by frameworks such as GRI, SASB, and WEFs metrics. This is an important shortcoming, as the importance of these issues and the risks they entail for both businesses and society are increasingly understood and accepted. 
Governance, broadly understood as relating both to company governance and society's governance, involves concerns about the power of Big Tech and problems associated with platforms and the abuse of monopoly power [53-55], and, for example, the polarizing effects of algorithmic filtering and social media $[56,57]$. Perhaps least emphasized by the AI ethics community has been the environmental dimension. However, recent developments suggest that these issues are attracting increasing attention. For example, issues related to the carbon footprint of using $\mathrm{AI}$ and Big Data to train large natural language models are now problematized $[8,49,58]$.

A primary reason for using the SDGs to evaluate $\mathrm{AI}$ is that they can help reconcile and bridge the potential gap that arises between business focused researchers and much of the AI ethics community. The former, at times, neglect important ethical challenges, while the latter sometimes neglects potentially important societal benefits because of the focus on the ethical challenges just discussed.

The SDGs force a broad perspective, and if AI is to be usefully evaluated in a compliance and reporting context, it is necessary to force the linkages between the E, S, and the $\mathrm{G}$. The beginnings of a framework that forces the linkages and encourages a balanced perspective of AI impacts is seen in Sætra [27]. In that article, the relationship between $\mathrm{AI}$ and the SDGs in general is being examined, and the current article builds on this, but focuses on its application in the context of business reporting and disclosure. Sætra [27] proposed that AI impacts should be evaluated in terms of direct and indirect impacts, and that an analytical framework that distinguishes between micro, meso and macro level effects is employed in order to foster both nuance and the ability to understand the broader and long term effects of AI [27]. This framework is shown Figure 3. If an AI system aimed at improving the efficiency of workers, for example, was introduced, this could conceivably have positive meso level impacts, as the profitability and growth of a particular business would be improved (SDG 8). However, a broader analysis based on the framework presented below might show that the system had negative effects on the conditions of the individual workers (negative micro level effects on SDG 8). Furthermore, such a system-if proprietary-could lead to increased inequalities (negative macro level impacts on SDG $10)$.

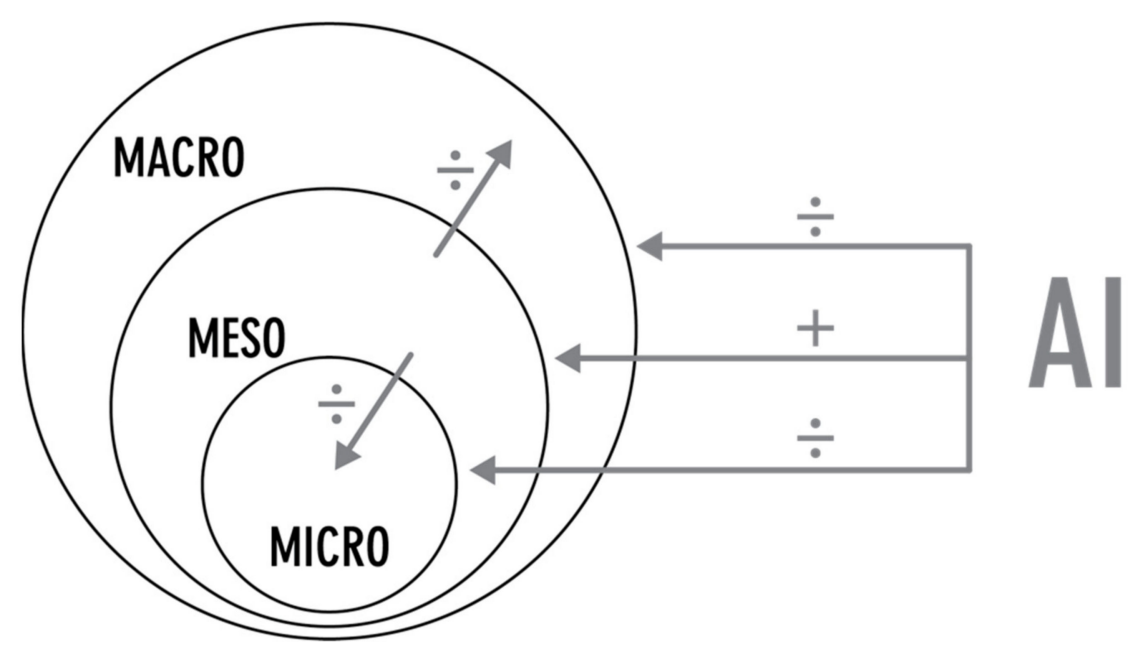

Figure 3. Three levels and the possibility of secondary effects, from Sætra [27].

\subsection{The Process and the Framework}

These considerations provide the basis for establishing the foundation of a framework for evaluating the sustainability related impacts of AI, and a general outline of the framework can now be established, before its potential usefulness is shown through an examination of the case of Microsoft's sustainability reporting. 
Starting with one of the key elements in the framework, Figure 4 shows how the impacts for any of the SDGs can be summarized, here exemplified with SDG 8, and with sample guide questions related to this goal. The core of the framework is the complete set of such tables, in which the key guide questions and most probable interlinkages are already present in order to guide the evaluation for a specific company. The guide questions are established by mapping the issues examined in, for example, the literature of AI ethics to the various SDGs and the micro, meso, and macro levels. A wide array of sources mentioned previously in this article will be helpful in establishing these questions, and it will also be possible to develop a minimal, intermediate, and comprehensive set of questions, and also to tailor the questions to particular types of industries or applications of AI.

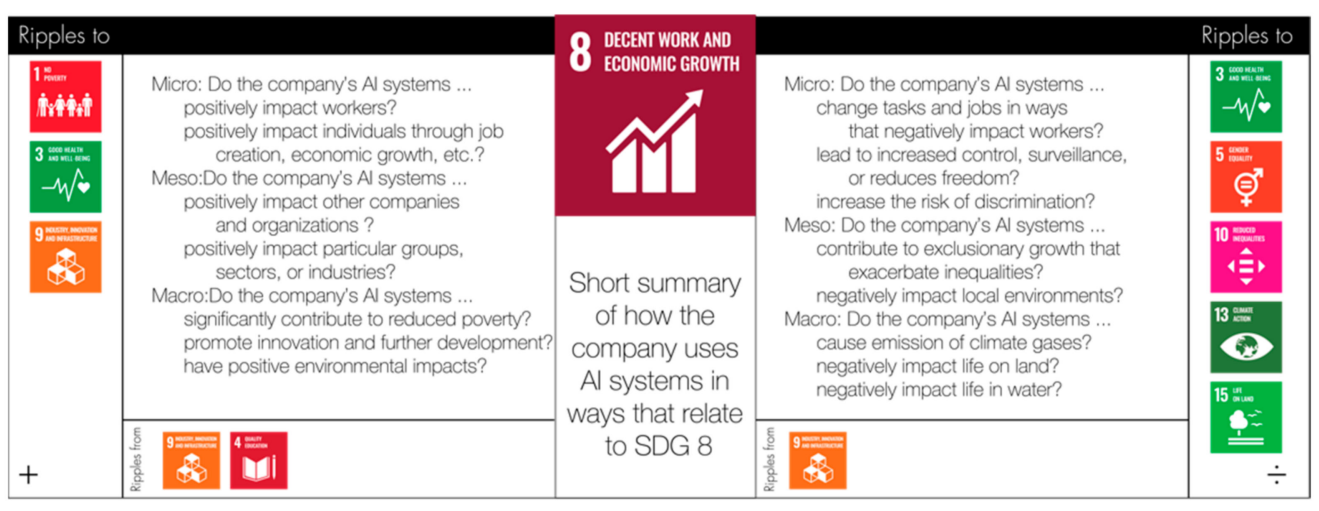

Figure 4. Table showing the key elements in the framework.

In the center, under the SDG 8 symbol, a brief summary of total impact will be provided. To the left of the center, all positive impacts are summarized and presented. In the context of SDG 8, the guide questions relate to how AI systems impact individuals and workers on the micro level, for example in terms of AI systems that monitor adherence to workers' rights and access to decent work. On the meso level, positive impacts on business growth on the business, region, or sector level are examined, but also whether particular groups of people (i.e., minorities) are positively affected by the economic growth of access to work. On the macro level, the contribution to (sustainable) economic growth is vitally important, and thus also how these impacts relate to other goals.

If the AI system contributes to economic growth that reduces poverty, provides improvements of health, innovation and infrastructure, for example, these SDGs are listed in the far left "Ripples to" column, in order to highlight interlinkages between economic growth, decent work and these other goals. In the bottom cell- "Ripples from"-the company's impact on other SDGs that have indirect effects on SDG 8 are listed (here SDG 4 and 9 are used as examples). The right side of the figure summarizes and structures the negative impacts related to SDG 8 . The guide questions might here focus on increased worker surveillance and changes in work due to automation (micro), increased difference between groups and exclusionary growth (meso), and climate gas emissions resulting from the systems used (macro). These effects are related to a range of SDGs, and the potential negative indirect effects to SDGs 3, 5, 10,13 and 15 are listed as an example.

The complete tables with guide questions are currently being developed, and the exact details of the table are not the key concern in this article. Instead, the general principle for approaching AI systems and their sustainability impacts is shown through this example. Any researcher or sustainability officer working on a sustainability report can build on this framework in order to structure their analysis and communication regarding these issues.

The complete framework can be adapted to the different needs of different businesses, and while the table above could be presented directly in the sustainability report, it could also be attached in appendices or be made available online. It need not even be published at 
all, but its results could be summarized in text form. However, the benefits of a structured approach to the impacts of AI could easily be lost with such an approach.

A different question briefly broached above is what sort of process surrounds the use of this framework. Once again, the framework is amenable to a wide range of approaches, but one standard approach often used when developing a sustainability strategy and reporting approach is to start with a situation analysis followed by a materiality analysis. The situation analysis typically consists of internal and external interviews, a SWOT analysis, and a literature review, which in this case will focus specifically on AI related sustainability impacts. Next, a materiality analysis will allow the company to discover which of the SDGs (a) their stakeholders are particularly concerned about, and (b) they themselves impact most directly and to which they have the ability to adjust their impacts. Such a materiality analysis would allow the company to single out a selection of SDGs of particular importance, and thus allow them to forego a full analysis of all 17 SDGs.

\subsection{AI Evaluation in Practice}

In order to show how this framework can be used to improve the analysis and reporting of AI impacts, an example of actual ESG reporting is helpful. Microsoft (MS) is here used as an example of how sustainability impacts can be evaluated and disclosed. MS is selected as a case because it represents one of the larger companies in the sector and the author is familiar with their sustainability reporting. Other comparable companies (Big Tech) have been examined to ensure that MS is not particularly bad or good at sustainability reporting, and MS thus serves as a useful example of how AI impacts have been discussed, and how the framework here described might improve reporting on AI impacts. MS in itself is not of particular interest, and any comparable company could have been used to illustrate the findings here produced. The company is a key developer of AI systems, and their Azure platform is both used by MS itself and by clients in a myriad of ways. MS releases a large number of documents related to CSR and ESG related activities, but their yearly CSR reports for 2018 and 2019 are here used as the basis for the discussion. The reports are called CSR reports, but they could also be referred to as sustainability reports. However, these reports also contain links to and descriptions of other reports, which will be of interest for those inclined to pursue a full and comprehensive analysis of MS's efforts. For this example, the main purpose is to examine to what extent $\mathrm{AI}$ is discussed in relation to CSR and ESG, whether or not the SDGs are used and/or discussed, and if so: how they are discussed.

First of all, the alphabet soup discussed above is also reflected in MS's efforts to demonstrate their CSR and ESG efforts. Their online list of awards and recognitions lists 13 different sources, including FTSE4Good, The Carbon Disclosure Project, and Sustainalytics [59], and their 2018 CSR report also mentions adherence to the GRI, RAFI (UN Guiding Principles on Business and Human Rights Reporting Framework), and the UN Global Compact [60]. CSR reporting is stated to be based on a need to create trust between the company, customers, and partners [61], and these efforts are often assumed to be related to the basic idea that companies rely on such trust and a social license to operate [10].

As mentioned, there is a tendency to discuss the positive impact of $\mathrm{AI}$ in terms of examples of use cases, such as the use of Azure in order to improve energy efficiency in the United Arab Emirates, Marriot using AI systems to improve their water, carbon, and land change footprint, and using Azure's optical analysis functions to analyze the development of arctic glaciers. These are all examples of potential benefits from AI, and throughout the report discussing MS's contribution to the SDGs, technology is said to be an important driver of change, and examples of beneficial use are provided [42]. These examples could easily be implemented in the framework presented in Section 4.1, and instead of being presented as isolated stories, various positive and negative effects could be presented as a whole, enabling any stakeholder to more easily assess the overall AI related impacts.

MS's 2018 CSR report only superficially addresses the SDGs, and the same goes for the 2019 version, even if there, they use the SDG symbols in the chapter title pages $[60,61]$. 
However, the 2019 report refers to their sustainability website [62], and MS later released the separate report Microsoft and the United Nations Sustainable Development Goals [42], which deals in detail with MS's SDG related efforts. The following describes how AI impacts are discussed in these three reports.

Starting with the 2018 report [60], MS emphasizes how just about all their major products are AI infused, and thus opens the door for a broad evaluation of the impacts of AI. First of all, their products reach most people in modern societies and are an integral part of the modern workplace, in which MS 365 plays an important part in the highly visible front end, while other MS systems play important roles in the back end. Azure is the core of the back end system, and this is the core of MS's AI and the source of the infusion experienced in most or all other MS products. Dynamics 365 is key for the business applications, and it is also described as AI driven. They describe how Azure lets all their clients build AI and get the most out of their data, and that they are "democratizing data science and AI" with Azure [60].

MS states that they are leading in AI research, and that their goal is to facilitate customer adoption and innovation in all aspects of business and society. AI will, they state, create "new opportunities for education and healthcare, address poverty, and achieve a more sustainable future" [60]. They state that they champion ethical AI, and at one point they explicitly address a concern related to bias in AI systems:

And, as we make advancements in AI, we are asking ourselves tough questionslike not only what computers can do, but what should they do. That's why we are investing in tools for detecting and addressing bias in AI systems and advocating for thoughtful government regulation. [60] (p. 12)

However, there is no description of the current status or plan for action for this work. They also state that AI "raises complex ethical questions about the impact on society including jobs, privacy, inclusiveness, and fairness" [60] (p. 41), and point to their book The Future Computed where AI related challenges are mentioned. In this book they discuss the role of AI in society and identify six ethical principles for the development and use of AI: fairness, reliability and safety, privacy and security, inclusivity, transparency, and accountability [63]. The above concerns and principles would benefit from being analyzed and discussed in relation to the relevant SDGs, as this would allow for a more holistic account of the impacts of MS's activities, rather than isolated discussions in which concerns and strengths are discussed without being related to the overall ESG impacts.

Similarly, they highlight the need for AI systems to be accessible, and point to work with governments, private sector and nonprofit organizations and how they donated $\$ 1,4$ billion in software that year. Furthermore, they highlight their AI for Earth and AI for Accessibility programs-“"putting AI tools into the hands of change-makers" [60] (p. 13). Finally, they have partnered with nonprofit organizations to provide computer science learning experiences for "millions of young people around the world" [60] (p. 13). All these issues are highly relevant for the SDGs, as many of the goals refer to the need for universal, equal, and affordable access to new technologies and innovations, and also to nondiscrimination [27]. However, the report makes no efforts to systematically evaluate the impact of AI and link these to the SDGs, and any negative impacts are mainly glimpsed through the short descriptions of philanthropic activity and vague references to investments in antibias solutions and advocacy for government regulation.

In the 2019 report, much of the same positive potential is highlighted through examples [61]. In this report, they refer to a new book named Tools and Weapons: The Promise and the Peril of the Digital Age [64], in which two MS directors discuss the potential challenges of AI. The six AI principles mentioned above are this year included and briefly explained, and "AI for good" is the umbrella term for a wide range of "AI for ... " initiatives, which, in addition to the ones mentioned in the 2018 version, now includes AI for Humanitarian Action and AI for Cultural Heritage. The separate chapter on AI begins with the following statement: "We build AI responsibly, taking a principled approach to guide the development and use of artificial intelligence with people at the center of everything we do" [61]. 
While the CSR reports seemingly acknowledge that there are issues related to the ethical challenges of AI, there is very little in terms of discussion about the specifics of the potential harms originating in MS AI systems, and no discussion about the potential risks to MS as a company resulting from neglecting such impacts.

The report focusing specifically on MS and the SDGs is particularly telling, in that it clearly states that it is about how MS contributes to the SDGs, and that it is not an effort to evaluate MS's overall impact on the goals. Technology, it states:

... is a powerful force for good, and all of us here at Microsoft are working together to foster a sustainable future where everyone has access to the benefits it provides and the opportunities it creates. [42] (p. 4)

While the use of examples and sunshine stories about AI impacts, as seen in the MS reports, are not erroneous, they can be considered to be incomplete without a deeper and more comprehensive analysis of the ESG related risks of AI. For example, while the MS and SDG report highlights MS's efforts to use AI to identify and counter deepfakes, it makes no mention of how AI systems are first used to create deepfakes. Similarly, while AI systems can help conservation efforts by analyzing and tracking various animal species, for example, the same technology can be used by those who wish to hunt those same species. This is a general problem often neglected in the analysis of AI impacts. The fact that $\mathrm{AI}$ is a double edged sword, and that a lot of use cases demonstrating positive impacts simultaneously provides examples of how to use AI for bad, must be remembered when AI impact is described [27]. This is where the framework presented in this article helps structure and present the various effects of AI.

AI does indeed have great potential, but in order to provide stakeholders with actionable insight into the real threats and opportunities companies using AI systems face, there is a need for connecting ESG reporting more closely to AI ethics research. This, in turn, can be achieved by using the SDGs as the foundation of the analysis of AI impacts, linkages between the different SDGs, for seeing impacts at the micro, meso, and macro level in its totality, and distinguishing between direct and indirect effects [27]. The separation of effects into different levels would allow MS to show how their software has both positive and negative impacts on workers, as they link this to SDG 8 (decent work and economic growth). In the initial analysis, they might describe how their entire business suite allows for convenience and productivity for workers, and cost savings and more effective management for employers. These micro level effects can be presented as positive, and so could the meso level effects related to increased company profitability, and the macro level effects related to potential economic growth, the other aspect of SDG 8. Furthermore, their AI systems can be used to improve infrastructure and for research and innovation purposes (SDG 9), which, again, foster economic growth and demonstrate the linkages between the various goals.

However, such an analysis is incomplete without a discussion of how employers might use the 365 suite as a tool of surveillance and control, thus causing potential negative micro level effects for employees at the same time [65]. Similarly, their investments in anti-bias solutions indicate that they are well aware of how current AI systems are prone to bias due to a variety of sources (often related to data), and this could be accompanied by an acknowledgement that AI might have negative impacts on, for example, SDG 5, related to gender equality. However, in this context the fact that AI might also reduce bias, as humans are also prone to bias, would reasonably be included.

As has become apparent in the preceding paragraphs, AI has a wide range of potential impacts, but the overall impact is exceedingly hard to evaluate if the different impacts are presented as isolated use cases spread throughout a long sustainability report. By applying the framework here developed, we have already seen how SDG 8, for example, could be presented in the form of the table in Figure 4. We have also seen that MS has described positive AI related impacts to the monitoring of glaciers, but with the framework here proposed, such impacts would be presented along with a discussion of how the use of AI also produces emissions that ultimately leads to glacier melt and climate change. In 
addition to increased transparency and honesty, which is itself beneficial, the framework is conducive to an approach in which trade offs are automatically part of any consideration of the sustainability of AI. This is because benefits and downsides are presented side by side, and in relation to each other. An example is how AI can be used to reduce human bias, while we also know that AI systems can themselves be biased in ways that are difficult to uncover. Education is another field in which MS promotes the positive impacts of AI, but we have also recently seen that the European Commission has labelled the use of AI in school a high risk application [66].

Similar considerations related to all the SDGs are presented in more detail in Sætra [27]. The next step following the work here presented is the development of the full framework built on the principles here described, based on a wide range of sources that describe various AI related sustainability impacts. By using the SDGs more actively in their ESG accounting and reporting, companies might still be able to "greenwash" and be tempted to use the SDGs more as window dressing that simply structures attempts to portray how well the company does. However, as this article has shown, ESG reporting is fundamentally about realistically communicating the positive and negative impacts of a company's activities, and the demands-both from regulators and other stakeholders-for honest and nuanced data and analyses are growing stronger by the day. The framework here presented provides an easy to use approach that forces some structure and demands a consideration of the trade offs involved in using AI systems, while it also allows the business community to make use of the knowledge produced in academia.

\section{Conclusions}

As AI systems permeate modern societies, a growing need to account for and understand their impacts emerge. This article has discussed various aspects related to how companies report on and disclose ESG related impacts, with a particular focus on how AI impacts might be accounted for in this context. This is increasingly important as companies of all kinds either build and develop or implement AI systems as elements in their overall activities.

One particular framework for analyzing and describing AI impacts is the SDGs. This framework has been adopted by a large number of companies, but it is usually not used in a systematic manner. This article has used MS as an example of how the SDGs are partly used in CSR and ESG reporting, but shows that there is great potential for a deeper and more comprehensive use of the SDGs. The article has also shown how to use the SDGs to analyze activities involving AI systems, with a particular emphasis on the need to go beyond simplistic analyses of how AI might relate superficially to each of the SDGs individually. For the SDGs to become a meaningful tool for analyzing AI system impacts, the interdependence between the SDGs must be factored in, and it is also beneficial to distinguish between the micro, meso, and macro level effects [27].

This article has presented the foundation of a framework to address the major challenges businesses experience related to evaluating and reporting on AI related sustainability impacts. It is not as of yet a complete framework, but while the complete framework is already under development, the core ideas here presented also allow any researcher or sustainability officer to start using the basic ideas in their own work, or to build on this and develop their own frameworks for specific (or general) purposes.

Improving the understanding of and reporting on ESG related AI impacts through the proposed framework is important for two reasons. First of all, companies might be said to have a responsibility to cause no harm, and in order to avoid causing harm they must thoroughly understand the impacts of their actions. For some companies, using the framework here proposed could lead to a deeper understanding of the sustainability of their actions. Where such an understanding has been lacking, this could in itself lead to changes in activity. Other companies might already be aware of most of their ESG related impacts, but a framework that demands more transparency and deeper analyses will potentially also affect these. While general reporting frameworks will not require 
that companies disclose indirect effects and broader societal and economic effects, the framework here presented requires this. This will both deepen the understanding of the impacts and make it harder for companies to sweep the negative impacts under the rug.

Secondly, a wide range of stakeholders are becoming increasingly aware of the problems highlighted in AI ethics, and any company that does not sufficiently align their businesses with this knowledge risks jeopardizing their social license to operate. This means that even if the executives of a company themselves do not care that much about their negative impacts, stakeholders do, and will increasingly punish companies that do not perform their best to mitigate adverse impacts. Lackluster ESG performance will make capital harder to come by, it will impede the goal of attracting the most competent workers, and it will hurt the company's relation with both suppliers and customers.

One might argue that Big Tech is too big to be influenced by better frameworks for the evaluation and disclosure of AI impacts. However, the preceding considerations suggest that even such companies will be affected by an increased understanding of their negative ESG related impacts. One advantage of the framework here presented is that such an increased understanding might occur even if the larger technology companies themselves did not take it particularly seriously. The framework is generic and aimed at highlighting general ESG related impacts of AI systems, and as soon as some companies start using it, this will engender increased understanding of the impacts even of those that do not use the framework. This could lead to negative reactions from both capital markets and consumers towards those that are seen not to take these issues seriously. Even more important, perhaps, is that this will provide regulators with a better understanding of the impacts of AI systems, and it is evident from recent developments in Europe that there is an increased willingness to regulate both data and the use of AI systems more generally. Not even big tech companies are immune to the collective pressure of markets, customers, and regulators.

Funding: This research received no external funding.

Institutional Review Board Statement: Not applicable.

Informed Consent Statement: Not applicable.

Data Availability Statement: Not applicable.

Conflicts of Interest: The author declares no conflict of interest.

\section{References}

1. Makridakis, S. The forthcoming Artificial Intelligence (AI) revolution: Its impact on society and firms. Futures 2017, 90, 46-60. [CrossRef]

2. De Sousa, W.G.; de Melo, E.R.P.; Bermejo, P.H.D.S.; Farias, R.A.S.; Gomes, A.O. How and where is artificial intelligence in the public sector going? A literature review and research agenda. Gov. Inf. Q. 2019, 36, 101392. [CrossRef]

3. Di Vaio, A.; Palladino, R.; Hassan, R.; Escobar, O. Artificial intelligence and business models in the sustainable development goals perspective: A systematic literature review. J. Bus. Res. 2020, 121, 283-314. [CrossRef]

4. Brynjolfsson, E.; Mcafee, A. The business of artificial intelligence. Harv. Bus. Rev. 2017, 7, 3-11.

5. Walker, J.; Pekmezovic, A.; Walker, G. Sustainable Development Goals: Harnessing Business to Achieve the SDGs through Finance, Technology and Law Reform; John Wiley \& Sons: Hoboken, NJ, USA, 2019.

6. Verbin, I. Corporate Responsibility in the Digital Age: A Practitioner's Roadmap for Corporate Responsibility in the Digital Age; Routledge: London, UK, 2020.

7. United Nations. Transforming Our World: The 2030 Agenda for Sustainable Development; Division for Sustainable Development Goals: New York, NY, USA, 2015.

8. Van Wynsberghe, A. Sustainable AI: AI for sustainability and the sustainability of AI. AI Ethics 2021, 1-6. [CrossRef]

9. Brundtland, G.H.; Khalid, M.; Agnelli, S.; Al-Athel, S.; Chidzero, B. Our Common Future; Oxford University Press: New York, NY, USA, 1987; Volume 8.

10. Demuijnck, G.; Fasterling, B. The social license to operate. J. Bus. Ethics 2016, 136, 675-685. [CrossRef]

11. Moon, J. Corporate Social Responsibility: A Very Short Introduction; OUP Oxford: Oxford, UK, 2014.

12. Marczewska, M.; Kostrzewski, M. Sustainable business models: A bibliometric performance analysis. Energies 2020, 13, 6062. [CrossRef] 
13. Nosratabadi, S.; Mosavi, A.; Shamshirband, S.; Kazimieras Zavadskas, E.; Rakotonirainy, A.; Chau, K.W. Sustainable business models: A review. Sustainability 2019, 11, 1663. [CrossRef]

14. Jones, T.M. Corporate social responsibility revisited, redefined. Calif. Manag. Rev. 1980, 22, 59-67. [CrossRef]

15. Petit, N. Big Tech and the Digital Economy: The Moligopoly Scenario; Oxford University Press: Oxford, UK, $2020 ;$ p. 11.

16. Zuboff, S. The Age of Surveillance Capitalism: The Fight for a Human Future at the New Frontier of Power: Barack Obama's Books of 2019; PublicAffairs: New York, NY, USA, 2019.

17. Vinuesa, R.; Azizpour, H.; Leite, I.; Balaam, M.; Dignum, V.; Domisch, S.; Felländer, A.; Langhans, S.D.; Tegmark, M.; Nerini, F.F. The role of artificial intelligence in achieving the Sustainable Development Goals. Nat. Commun. 2020, 11, 1-10. [CrossRef] [PubMed]

18. Berenberg. Understanding the SDGs in Sustainable Investing; Joh Berenberg, Gossler \& Co. KG: Hamburg, Germany, 2018.

19. Esty, D.C.; Cort, T. (Eds.) Values at Work: Sustainable Investing and ESG Reporting; Palgrave McMillan: Cham, Switzerland, 2020.

20. Eckhart, M. Financial Regulations and ESG Investing: Looking Back and Forward. In Values at Work; Esty, D.C., Cort, T., Eds.; Palgrave McMillan: Cham, Switzerland, 2020; pp. 211-228.

21. European Commision. The European Green Deal; European Commision: Geneva, Switzerland, 2019.

22. EU Technical Expert Group on Sustainable Finance. Taxonomy: Final Report of the Technical Expert Group on Sustainable Finance; EU Technical Expert Group on Sustainable Finance: Brussels, Belgium, 2020.

23. Bose, S. Evolution of ESG Reporting Frameworks. In Values at Work; Esty, D.C., Cort, T., Eds.; Palgrave McMillan: Cham, Switzerland, 2020; pp. 13-33.

24. World Economic Forum. Measuring Stakeholder Capitalism: Towards Common Metrics and Consistent Reporting of Sustainable Value Creation; World Economic Forum: Graubunden, Switzerland, 2020.

25. SDG Compass. SDG Compass: The Guide for Business Action on the SDGs; Global Reporting Initiative: Amsterdam, The Netherlands, 2015.

26. Chui, M.; Harryson, M.; Manyika, J.; Roberts, R.; Chung, R.; van Heteren, A.; Nel, P. Notes from the AI Frontier: Applying AI for Social Good; McKinsey Global Institute: San Francisco, CA, USA, 2018.

27. Sætra, H.S. AI in context and the sustainable development goals: Factoring in the unsustainability of the sociotechnical system. Sustainability 2021, 13, 1738. [CrossRef]

28. Truby, J. Governing Artificial Intelligence to benefit the UN Sustainable Development Goals. Sustain. Dev. 2020, 28, 946-959. [CrossRef]

29. Khakurel, J.; Penzenstadler, B.; Porras, J.; Knutas, A.; Zhang, W. The rise of artificial intelligence under the lens of sustainability. Technologies 2018, 6, 100. [CrossRef]

30. Toniolo, K.; Masiero, E.; Massaro, M.; Bagnoli, C. Sustainable business models and artificial intelligence: Opportunities and challenges. In Knowledge, People, and Digital Transformation; Springer: Cham, Switzerland, 2020; pp. $103-117$.

31. Yigitcanlar, T.; Cugurullo, F. The sustainability of artificial intelligence: An urbanistic viewpoint from the lens of smart and sustainable cities. Sustainability 2020, 12, 8548. [CrossRef]

32. Dignum, V. Responsible Artificial Intelligence: How to Develop and Use AI in a Responsible Way; Springer: Cham, Switzerland, 2019.

33. Floridi, L.; Cowls, J.; Beltrametti, M.; Chatila, R.; Chazerand, P.; Dignum, V.; Luetge, C.; Madelin, R.; Pagallo, U.; Rossi, F. AI4People-An ethical framework for a good AI society: Opportunities, risks, principles, and recommendations. Minds Mach. 2018, 28, 689-707. [CrossRef] [PubMed]

34. ITU. AI4Good Global Summit. Available online: https:/ /aiforgood.itu.int (accessed on 31 January 2021).

35. Tomašev, N.; Cornebise, J.; Hutter, F.; Mohamed, S.; Picciariello, A.; Connelly, B.; Belgrave, D.C.; Ezer, D.; van der Haert, F.C.; Mugisha, F. AI for social good: Unlocking the opportunity for positive impact. Nat. Commun. 2020, 11, 1-6. [CrossRef]

36. Google. AI for Social Good: Applying AI to Some of the World's Biggest Challenges. Available online: https://ai.google/socialgood/ (accessed on 20 February 2021).

37. Berberich, N.; Nishida, T.; Suzuki, S. Harmonizing Artificial Intelligence for Social Good. Philos. Technol. 2020, 33, 613-638. [CrossRef]

38. GRI. Consolidated Set of GRI Sustainability Reporting Standards 2020; GRI: Amsterdam, The Netherland, 2020.

39. GRI. Linking the SDGs and the GRI Standards; GRI: Amsterdam, The Netherland, 2020.

40. Nižetić, S.; Djilali, N.; Papadopoulos, A.; Rodrigues, J.J. Smart technologies for promotion of energy efficiency, utilization of sustainable resources and waste management. J. Clean. Prod. 2019, 231, 565-591. [CrossRef]

41. Kaab, A.; Sharifi, M.; Mobli, H.; Nabavi-Pelesaraei, A.; Chau, K.-w. Combined life cycle assessment and artificial intelligence for prediction of output energy and environmental impacts of sugarcane production. Sci. Total Environ. 2019, 664, 1005-1019. [CrossRef]

42. Microsoft. Microsoft and the United Nations Sustainable Development Goals; Microsoft: Redmond, WA, USA, 2020.

43. Google. Environmental Report 2019; Google: Menlo Park, CA, USA, 2019.

44. Google. Working Together to Apply AI for Social Good. Available online: https://ai.google/social-good/impact-challenge (accessed on 23 February 2021).

45. Nilashi, M.; Rupani, P.F.; Rupani, M.M.; Kamyab, H.; Shao, W.; Ahmadi, H.; Rashid, T.A.; Aljojo, N. Measuring sustainability through ecological sustainability and human sustainability: A machine learning approach. J. Clean. Prod. 2019, $240,118162$. [CrossRef] 
46. Houser, K.A. Can AI Solve the Diversity Problem in the Tech Industry: Mitigating Noise and Bias in Employment Decision-Making. Stanf. Technol. Law Rev. 2019, 22, 290.

47. Noble, S.U. Algorithms of Oppression: How Search Engines Reinforce Racism; New York University Press: New York, NY, USA, 2018.

48. Buolamwini, J.; Gebru, T. Gender shades: Intersectional accuracy disparities in commercial gender classification. In Proceedings of the Conference on Fairness, Accountability and Transparency, New York, NY, USA, 23-24 February 2018; pp. 77-91.

49. Bender, E.M.; Gebru, T.; McMillan-Major, A.; Shmitchell, S. On the dangers of stochastic parrots: Can language models be too big. In Proceedings of the 2021 ACM Conference on Fairness, Accountability, and Transparency, Virtual Event Canada, 3-10 March 2021. [CrossRef]

50. Solove, D.J. Privacy and power: Computer databases and metaphors for information privacy. Stanf. Technol. Law Rev. 2000, 53, 1393. [CrossRef]

51. Yeung, K. 'Hypernudge': Big Data as a mode of regulation by design. Inf. Commun. Soc. 2017, 20, 118-136. [CrossRef]

52. Sætra, H.S. When nudge comes to shove: Liberty and nudging in the era of big data. Technol. Soc. 2019, 59, 101130. [CrossRef]

53. Culpepper, P.D.; Thelen, K. Are we all amazon primed? consumers and the politics of platform power. Comp. Political Stud. 2020, 53, 288-318. [CrossRef]

54. Gillespie, T. The politics of 'platforms'. New Media Soc. 2010, 12, 347-364. [CrossRef]

55. Sagers, C. Antitrust and Tech Monopoly: A General Introduction to Competition Problems in Big Data Platforms: Testimony Before the Committee on the Judiciary of the Ohio Senate. Available online: https://papers.ssrn.com/sol3/papers.cfm?abstract_ $\mathrm{id}=3471823$ (accessed on 17 October 2019).

56. Sætra, H.S. The tyranny of perceived opinion: Freedom and information in the era of big data. Technol. Soc. 2019, 59, 101155. [CrossRef]

57. Sunstein, C.R. \# Republic: Divided Democracy in the Age of Social Media; Princeton University Press: Princeton, NJ, USA, 2018.

58. Brevini, B. Black boxes, not green: Mythologizing artificial intelligence and omitting the environment. Big Data Soc. 2020, 7 , 2053951720935141. [CrossRef]

59. Microsoft. Awards \& Recognition. Available online: https://www.microsoft.com/en-us/corporate-responsibility/recognition (accessed on 15 February 2021).

60. Microsoft. Microsoft 2018: Corporate Social Responsibility Report; Microsoft: Redmond, WA, USA, 2018.

61. Microsoft. Microsoft 2019: Corporate Social Responsibility Report; Microsoft: Redmond, WA, USA, 2019.

62. Microsoft. Our Commitment to Sustainable Development. Available online: https://www.microsoft.com/en-us/corporateresponsibility/un-sustainable-development-goals (accessed on 20 February 2021).

63. Microsoft. The Future Computed: Artificial Intelligence and Its Role in Soviety; Microsoft: Redmond, WA, USA, 2018.

64. Smith, B.; Browne, C.A. Tools and Weapons: The Promise and the Peril of the Digital Age; Penguin: New York, NY, USA, 2019.

65. Manokha, I. The Implications of Digital Employee Monitoring and People Analytics for Power Relations in the Workplace. Surveill. Soc. 2020, 18, 540-554. [CrossRef]

66. Europan Commision. Europe Fit for the Digital Age: Commission Proposes New Rules and Actions for Excellence and Trust in Artificial Intelligence; Europan Commision: Geneva, Switzerland, 2021. 